\title{
Capacity analysis of the selected track system in partially ordered space
}

\author{
Eligiusz Mieloszyk ${ }^{1}$, Anita Milewska ${ }^{1}$, and Stawomir Grulkowski ${ }^{1, *}$ \\ ${ }^{1}$ Gdansk University of Technology, Faculty of Civil and Environmental Engineering, Narutowicza Str. 11/12, 80-233 Gdańsk
}

\begin{abstract}
A proper location of the interval sections has significant impact on the traffic flow in the railway track network. This issue is critical during line modernization as well as when a new solution accounting for the traffic forecast at particular element of the railway track network is developed. However, the situation is more complex and more expensive for railway stations since improvement of the capacity requires critical organizational changes and additional investments. We have applied partially ordered linear spaces to study the capacity of a track system.
\end{abstract}

\section{Introduction}

Well-developed switch routes are crucial in the train stations with high freight traffic [1,2]. The traffic at the station can be 'scheduled' or 'shunting'. Therefore, the track system in the station should be designed to provide sufficient traffic capacity and flow. The most loaded switch or a pair of switches in the station head is a bottleneck of the station (critical element) [3].

The highest traffic is usually forecasted for the selected day from the week (month or year). The critical element of the station or region (Fig. 1) is highly loaded due to long passing time, and this load is not evenly distributed.

Selected day has to be divided into $n$ intervals with durations $d_{i}, i=1,2, \ldots, n$ in order to determine a peak interval related to the largest number of the scheduled train operations, shunting operations and free locomotive operations through the critical element. The duration of the peak interval depends on the type of station, its size, the type of the transported cargo, the complexity of freight processes, station electrification, uniformity distribution of the load due to the train, shunting and locomotive operations on the selected day. The peak interval can last 45, 6090 minutes or even longer. Let us assume that the duration of the peak interval is given by $t$.

In order to determine maximum permissible load of the critical element, we can use the following equation which is related to the critical time interval $t$ :

$$
Z=\left[\begin{array}{c}
N \\
1 \\
E
\end{array}\right] A T+Z_{z}
$$

$$
\begin{aligned}
\text { where } & {\left[\begin{array}{c}
N \\
1 \\
E
\end{array}\right] \text { is the matrix in which } } \\
N & =\left[\begin{array}{lllllllll}
n_{1} & n_{2} & \ldots & n_{k} & \bar{n}_{1} & \bar{n}_{2} & \ldots & \bar{n}_{l}
\end{array}\right] \\
1 & =\left[\begin{array}{llllllll}
1 & 1 & \ldots & 1 & 1 & 1 & \ldots & 1
\end{array}\right]
\end{aligned}
$$

and $E$ is the identity matrix of the order $k+l$, $A=\operatorname{diag}\left[\alpha_{i i}\right]$ is the matrix of non-negative weights of the order $k+l$.

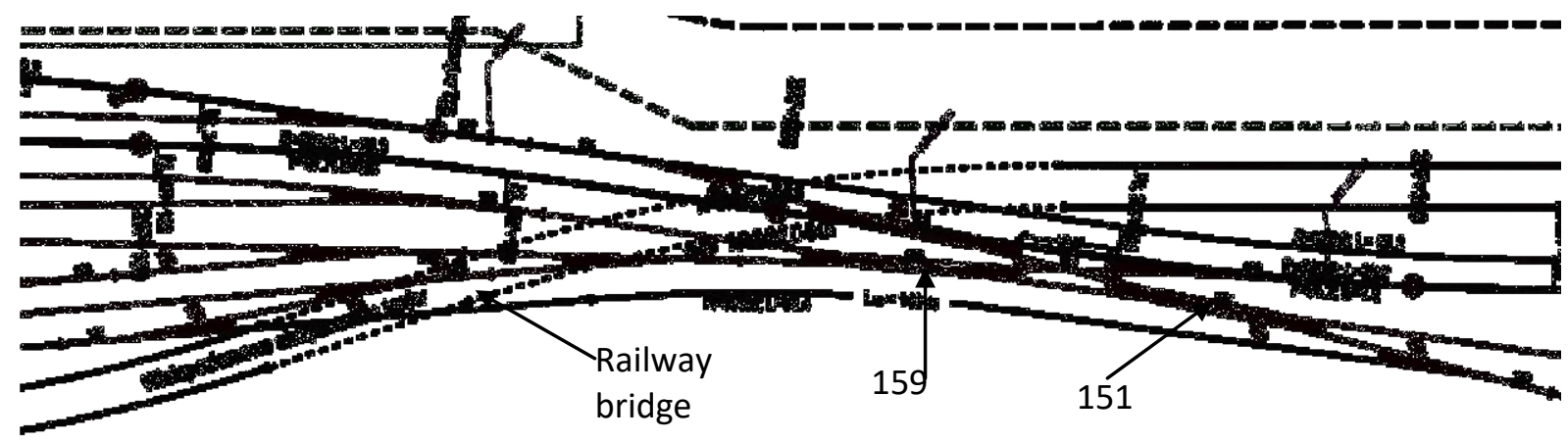

Fig. 1. Example of the head of the station with the critical elements - switch points \#151 and \#159.

\footnotetext{
* Corresponding author: slawomir.grulkowski@pg.edu.pl
} 
$Z_{Z}$ is a single-column matrix of disturbances with $k+l+2$ rows, and

$$
T=\left[\begin{array}{llllllll}
t_{1} & t_{2} & \ldots & t_{k} & \tau_{1} & \tau_{2} & \ldots & \tau_{l}
\end{array}\right]^{T}
$$

where []$^{T}$ is the transposition operation.

The quantities $n_{i}(i=1,2,3, \ldots, k), \bar{n}_{j}(j=1,2,3, \ldots, l)$ in the matrix $N$ indicate the number of freight trains and the number of shunting operations that contribute to the load of the critical element in the peak time interval, respectively.

The quantities $t_{i}(i=1,2,3, \ldots, k), \tau_{j}(j=1,2,3, \ldots, l)$ in the matrix $\boldsymbol{T}$ indicate the occupancy times of the critical element by the freight trains and by the shunting operations, respectively.

$Z$ can be called a generalized matrix of the occupancy of the critical element at the peak time interval.

\section{Partially ordered spaces for analysis of the capacity of some type of track systems}

Many quantities in different fields of engineering, medicine etc. are non-negative, which is a basis for the theory of positive systems [4]. The theory of positive systems uses a cone space rather than a linear space, making the description difficult and complex.

One can select a cone in the linear space X [5] and introduce a partial order $[6,7]$, which is given by the symbol $\geq$.

Definition 1:

The set $K \subset X$ is called a cone when the following conditions are met:

- $0 \in K$,

- if $x, y \in K$, then $x+y \in K$,

- $x \in K, \beta \in R_{+} \cup\{0\}$, then $\beta x \in K$,

- if $a \in K$ and $b \in K$ exists so that $b-n a \in K$ for $n$ $=1,2,3, \ldots$, then $a=0$,

- for each element $x \in X$ the elements $x_{1}, x_{2} \in K$ exist so that $x=x_{1}-x_{2}$.

Attention: The defined cone is not a geometrical concept but rather an abstract concept.

The definition of a partial order $\leq$ can be introduced based on the concept of the cone.

Definition 2:

$x \leq y$ then and only then when $y-x \in K$.

Linear space $X$ with the introduced partial order $\leq$ is called a partially ordered space, and the cone elements are called non-negative elements.

Let $X, Y$ be partially ordered spaces.

Definition 3:

Operation $U: X \rightarrow Y$ is non-negative then and only then, when $U x \geq 0$ for $x \geq 0$.

One can introduce the following definition of the partial order in the set of matrices (based on Definition 2).

* Corresponding author: slawomir.grulkowski@pg.edu.pl
Definition 4:

The matrix $B=\left[b_{i j}\right]_{\substack{0 \leq i \leq n \\ 0 \leq j \leq m}} \geq 0$ then and only then, when $\forall_{0 \leq i \leq n, 0 \leq j \leq m} b_{i j} \geq 0$.

Attention:

It is important to note that the matrix $e^{A t}$ is not always non-negative.

Conclusion 1:

The matrix $B \geq 0$ then and only then when all elements of $B$ are non-negative.

Properties of non-negative matrices:

- transposition of non-negative matrix is a nonnegative matrix,

- block matrix composed of non-negative matrices is a non-negative matrix,

- the sum of non-negative matrices is a non-negative matrix,

- the multiplication of non-negative matrices is a nonnegative matrix,

- every matrix can be expressed as a subtraction of two non-negative matrices.

In Def. 4 the matrices $N, 1, E$ and $A$ i $T$ are nonnegative. Based on the properties of non-negative matrix, the matrices $A T$ and $\left[\begin{array}{c}N \\ 1 \\ E\end{array}\right]$ are non-negative.

Conclusion 2.

Generalized matrix $Z$ of the occupancy of the critical element in the peak time interval is a non-negative matrix provided that the matrix of disturbances $Z_{Z}$ is non-negative.

Operation given by Eq. (1) is regarded as e.g. dependent on $T$ and it is a non-negative operation.

Using the matrix $Z$ given by Eq. (1), one can define the matrix

$$
Y=B Z+C
$$

where $B$ is a diagonal matrix composed of two blocks: the matrix $\left[\begin{array}{cc}-1 & 0 \\ 0 & -1\end{array}\right]$ and the identity matrix $E$ of the order $k+l$. The single-column matrix $C$ has $k+l+2$ rows, and the duration $t$ of the peak time interval is included in the first row. The second row contains the quantity $\bar{t}$ set by the designer and correlated with the weight interval $1 A T+c$ of the occupancy of the critical element in the peak interval. The constant $c$ is positive. The other elements of the matrix $C$ are zeros.

Conclusion 3.

The matrix $B$ can always be expressed as a subtraction of two non-negative matrices.

Using Eqs. (1) and (2), it is possible to determine a generalized closed dynamic system, as shown in Fig. 2. 


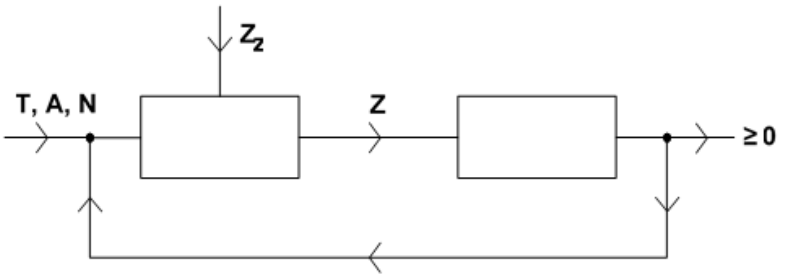

Fig. 2. Generalized closed dynamic system (non-negative)

The first block in the system in Fig. 2 operates according to Eq. (1) whereas the second block obeys Eq. (5). The feedback works in the case there is no nonnegative matrix at the output of the system. The construction process of the non-negative dynamic system is based on a mutual correction of the elements of input matrices $N, A$ and $T$ and the matrix of disturbances $Z_{Z}$. As an example, one can make the selected times $t_{i}$ from $i$ $=1,2, . ., k$ shorter by e.g. connecting the trains or by applying higher speeds.

This will change also $n_{i}(i=1,2,3, \ldots, k)$, and the weights etc. will be modified. It is possible to shorten $\tau_{j}(j=1,2,3, \ldots, l)$ in the same way by: using dualsystem locomotives, avoiding changing the head of the train, sufficiently long heads of the spur lines.

The examples shown here indicate opportunities offered by the method and possible applications.

\section{Application of the proposed method}

On September 11, 2017 during International Maritime Fair Baltexpo 2017, the agreement of the first project within the new budget perspective 2014-2020 has been signed to perform construction works. The project entitled "Extension of the turning basin No. 2" is realized within the investment project entitled "Deepening of the approach fairway and internal port basins of the Port of Gdynia - Stage I". The project includes modernization and extension of the turning basin \#2 (Fig. 3) along with the redevelopment of the pier No. III and the dredging works.

The activities will enable larger ships to enter the port since the diameter of the basin will be 80 meters larger and finally will achieve 480 meters. The existing turning basin of the diameter of 385 meters allows for turning the ships only up to 300 meters long. The size of the internal entrance to the port will achieve 140 meters (40 meters more than currently), and the depth of the fairway will be 16 meters (from 13.5 meters now). A new turning basin will accept larger ships, in particular the container ships of 400 meters long in 2018. A new container terminal will be developed, as well (Fig. 4).

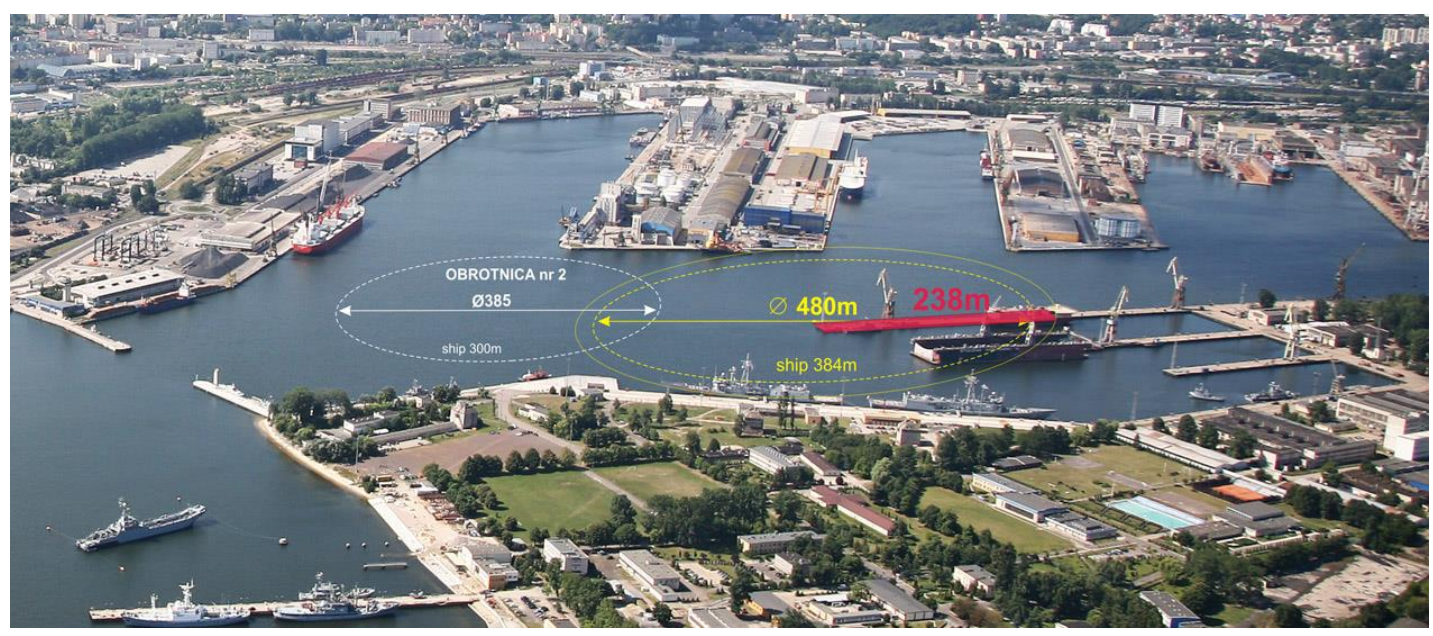

Fig. 3. Visualization of the concept of redevelopment of the turning basin No. 2 in the port of Gdynia. Credit: www.portalmorski.pl

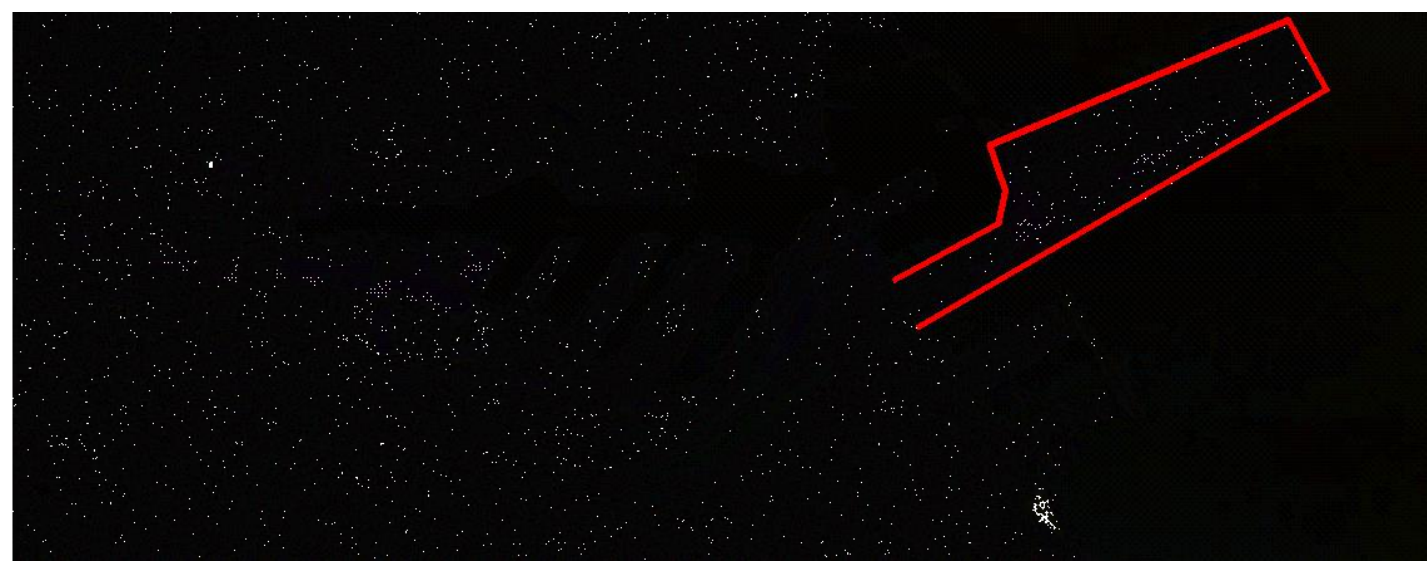

Fig. 4. The design of the container terminal in the External Port at the Port of Gdynia. Credit: www.port.gdynia.pl 
The investments enable significantly higher transshipment in the port of Gdynia, including the containers. As a result, the load and the traffic at the station Gdynia Port will be higher, which applies particularly to the switches at the station head located at the borders of the regions of railway traffic steering (bottlenecks, the highest load of the switch system). It has to be noted that the location of the wharves with respect to the main train lines at the station and the need to change the direction of movement generate a large number of the "contradictory operations" (in opposite directions) at the switch.

Currently, maximum 24 trains leaves the Gdynia Port station during the day. It is expected that the number of trains will be larger in the future $(50 \%$ more by 2025 , and dubled by 2045, including much heavier trains). This forecast depends on the global economy and the growth of the economy of Poland and neighbouring countries. It turns out that the operation of the Gdynia Port station should be adjusted to the situation and development of the Port of Gdynia itself. 2 .

We can use the dynamic closed system given in Fig.

We analyzed the draft designs of the switch headproposed within the task (workpackage) 'Improvement of the railway access to the Port of Gdynia'. The initial idea was to place the traffic directly through the switch (Fig. 1).

As shown in the scheme, the concept of the traffic between the regions of the Gdynia Port station is limited by the following aspects:

- the need to move the entire departure traffic from the regions located directly at the wharves and the Maritime Container Terminal towards classification tracks at the west side of the station - forecasted 48 trains/day by 2045 ;

- the need to perform arrival and transit traffic minimum 42 trains/day by 2045 ;

- shunting operations and the traffic due to the multiple units.

Lack of collision-free solutions will determine the risk and functional limitations of the system due to the need to make long distances between different parts of the station. It is necessary to remember that the occupancy duration of the critical element is calculated between the time of the decision of the train dispatcher regarding the route through the bottleneck and the time when the last car of the train leaves that route.

The maintenance of the switch system in the case of heavy traffic generates constantly high costs associated with the degradation of the parts of switches.

The example of the study of the reliability of the system is the analysis of the single slip switch \#151 (Fig. $5)$.

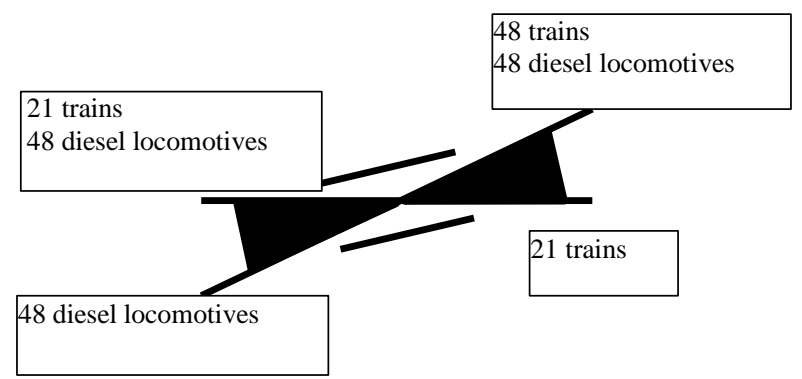

Fig. 5. Scheme of the switch \#151 with the traffic load

Table 1. Traffic load at the switch \#151

\begin{tabular}{|c|c|c|c|c|}
\hline Train type & 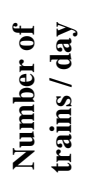 & 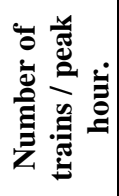 & 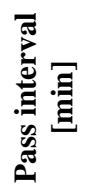 & 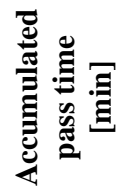 \\
\hline 1 & 2 & 3 & 4 & 5 \\
\hline Trains & 48 & 7 & 5 & 35 \\
\hline Locomotives & 48 & 7 & 2 & 14 \\
\hline Trains & 21 & 3 & 5 & 15 \\
\hline
\end{tabular}

Table 1 allows for the identification of the matrices introduced in Section 2. The elements of the matrix $N$ are given in the column 3. The elements of the matrix $T$ are given in column 4 . On the other hand, the matrix $A$ is the matrix of the weights of time. Each shunting or train operation described in this way can be influenced by the possible disturbances given by matrix $Z_{Z}$. All four matrices are corrected based on the feedback. This means that the elements of matrix can be influenced by the parameters such as the change of the type of switch, higher permissible speed, implementation of the centralized and automatic traffic control, dividing the sections into intervals, better locomotives, electrification etc. Separation of the traffic through designing train line intersections and station tracks at different levels is very effective. If the output from the system is the element from the cone in partially ordered space, the technical and technological changes are accepted (Fig. 2).

As a consequence of the lack of throughput of the switches \#151 and 159, we proposed to construct twolevel intersection (dashed lines in Fig. 1).

\section{Conclusions}

Partially ordered linear spaces and closed steering systems can be used to assess the occupancy of the track systems. This approach allows for planning the traffic at the station, forecasting the operation capacity, the development of a reliable system of the tracks, and increasing the station capacity in the case of higher transportation needs.

We used the proposed description and closed steering systems to analyze switch system at the Gdynia Port station. This enabled different solutions providing

\footnotetext{
Corresponding author: slawomir.grulkowski@pg.edu.pl
} 
reliability of the system, in which the development of the Port of Gdynia was taken into account.

\section{References}

1. S. Grulkowski, J. Zariczny, R. Gleba, Effect of different structure type traffic on railway line capacity, Pol. Marit. Research, 24, 82-88 (2017).

2. A. Massel, Design of train lines and train station), PKP Polskie Linie Kolejowe S. A., 2010 (in Polish).

3. R. Gleba, S. Grulkowski, D. Szczepiński, J. Zariczny, Significance of traffic analysis in the optimization process of railway infrastructure investment, Scient. and Technical Papers of the Associ of Engineers and Technicians of Communication in Krakow, 3, 63-82 (2016) (in Polish).

4. S. Rinaldi, L. Farina, I sistemi lineari positivi, Citta Studi Edizoni. 1995 (in Italian).

5. G. Strang, Introduction to Linear Algebra, Wellesley Cambridge Press, (2009).

6. E. Mieloszyk, Well defined solution problem for certain generalized boundary value problem, Studia Sci. Math. Hung., 32, 15-22 (1998).

7. E. Mieloszyk, Non-classical operational calculus in application to generalized dynamical systems, Polish Acad. of Sciences Scientific Publ., (2008). 